\title{
Male Urethral Malignant Neoplasm
}

National Cancer Institute

\section{Source}

National Cancer Institute. Male Urethral Malignant Neoplasm. NCI Thesaurus. Code C39867.

A primary or metastatic malignant neoplasm that affects the male urethra. 